\section{BMJ Open} Ophthalmology

\title{
Comparing the needs and preferences of patients with moderate and severe dry eye symptoms across four countries
}

\author{
Elisabeth Messmer, ${ }^{1}$ Colin Chan, ${ }^{2}$ Penny Asbell, ${ }^{3}$ Gary Johnson, ${ }^{4}$ Brigitte Sloesen, ${ }^{5}$ \\ Nigel Cook (iD ${ }^{5}$
}

To cite: Messmer E, Chan C Asbell P, et al. Comparing the needs and preferences of patients with moderate and severe dry eye symptoms across four countries. BMJ Open Ophthalmology 2019:4:e000360. doi:10.1136/ bmjophth-2019-000360

- Additional material is published online only. To view please visit the journal online (http://dx.doi.org/10.1136/ bmjophth-2019-000360).

These results have been presented as a poster at the HTAi Congress, Vancouver, 2 June 2018

Received 25 June 2019 Revised 2 0ctober 2019 Accepted 31 October 2019

Check for updates

(c) Author(s) (or their employer(s)) 2019. Re-use permitted under CC BY-NC. No commercial re-use. See rights and permissions. Published by BMJ.

${ }^{1}$ Department of Ophthalmology, Ludwig Maximilians University, Munich, Germany

${ }^{2}$ Central Clinical School, University of Sydney Sydney Medical School, Sydney, New South Wales, Australia

${ }^{3}$ Ophthalmology, Icahn School of Medicine at Mount Sinai, New York City, New York, USA ${ }^{4}$ Inpharmation, High Wycombe, UK

${ }^{5}$ Global Patient Access, Novartis Pharma AG, Basel, Switzerland

Correspondence to Dr Nigel Cook; nigel.cook@ novartis.com

\section{ABSTRACT}

Background/aims Understanding patients' perspectives of their disease can inform drug development and treatment decisions. In this study, we compared the preferences and needs of patients with moderate and severe dry eye symptoms from four different countries. Methods A quantitative questionnaire was developed based on the self-explicated conjoint methodology and was administered to 160 patients with moderate or severe dry eye disease (DED) from Australia, Germany, UK and the USA.

Results Patients with moderate dry eye symptoms ranked 'treatment satisfaction' as the most important aspect, whereas 'symptom bother' was more relevant for those in the severe group. Both the moderate and severe groups classified treatment effectiveness as the most important treatment attribute. This result was consistent across the four countries, although US patients gave significantly higher scores than patients from other countries $(p<0.001)$. Furthermore, patients from Australia ranked 'treatment experience' as significantly more important than the concern of side effects, whereas respondents from Germany exhibited the opposite trend ( $p<0.05$ for both). The health burden of DED is reflected in the average European Quality of Life-5 Dimensions 5 -level (EQ-5D) scores of 0.764 and 0.658 for patients with moderate and severe disease, respectively.

Conclusion Our results confirm that across the countries in the study, moderate and severe DED has a major impact on patients' quality of life and daily activities. By providing insight into the patient perspective of DED, our study helps identify outcomes that are important to patients and may guide future drug development and clinical decisionmaking.

\section{INTRODUCTION}

Dry eye disease (DED) is defined by the Tear Film and Ocular Surface Society's Dry Eye Workshop as 'a multifactorial disease of the ocular surface characterised by a loss of homeostasis of the tear film, and accompanied by ocular symptoms, in which tear film instability and hyperosmolarity, ocular surface inflammation and damage, and neurosensory abnormalities play etiological roles'. The medical management of DED continues to remain an unmet need, as expressed by

\section{Key messages}

What is already known about this subject?

- The medical management of dry eye disease continues to remain an unmet need, especially in patients with moderate and severe symptoms.

\section{What are the new findings?}

Herein, we compared the experience of disease among 160 patients with moderate and severe dry eye symptoms from different geographical regions (Australia, Germany, UK and the USA) and identified treatment attributes that are important to patients.

We found that 'treatment satisfaction' was the most important aspect for patients with moderate disease, whereas 'symptom bother' was more relevant for those in the severe group. However, both groups ranked treatment effectiveness as the most important treatment attribute.

\section{How might these results impact clinical}

\section{practice?}

Patient preference studies such as the present one have the potential to revolutionise clinical practice by allowing an individualised approach to the treatment of dry eye.

patients and physicians alike. ${ }^{2}{ }^{3}$ Only few novel treatments have emerged over the last decade despite a comparably large number of clinical trials. ${ }^{4}$ The availability and use of dry eye therapies in everyday practice vary across countries: ocular anti-inflammatory agents (cyclosporine A, lifitegrast) have been used intensively in the USA for several years, while in Australia lifitegrast was granted approval in early 2019; artificial tears containing hyaluronic acid are widely used in Europe, especially in Germany, but are not available in the USA.

Moderate and severe DED is associated with pain, limitation in daily activities, reduced vitality, poor general health and often depression. ${ }^{1}$ The impact of severe DED on patients' quality of life (QoL) was found to be comparable to that of angina. ${ }^{5}$ Patients with moderate and severe forms of the disease are 
also greatly affected in the workplace, where their dry eye symptoms can lead to a significant loss of productivity. ${ }^{6}$

In DED as in other diseases patient preference research has the potential to revolutionise healthcare practice by allowing an individualised approach to treatment in accordance with the needs expressed by the patient. ${ }^{7}$ When conducted early in the product development lifecycle, patient preference studies can inform the design of clinical trials (eg, through the inclusion of patientreported outcomes (PROs)), ensuring that outcomes that matter to patients are captured. ${ }^{8}$ With an increasing trend towards capturing relevant PROs in clinical trials, patient-centric information becomes an important input to value frameworks for evaluating new therapeutic interventions, to ensure they deliver value to the patient. ${ }^{8}$ This view is echoed in patient surveys ${ }^{9}$ and ongoing, largescale, multidisciplinary initiatives such as the patient preferences in benefit and risk assessments during the treatment life cycle (IMI PREFER) project, which is exploring ways to incorporate patient preference research into drug development at all stages of the product lifecycle. ${ }^{10}$ Evidence is accumulating on the utility of patient preference research in the regulatory decision-making process. ${ }^{11}$ However, despite the positive perception from regulatory agencies and stakeholders, examples on how best to incorporate patient preferences in therapeutic decision-making, in particular data obtained early in the product development lifecycle, remains sparse. ${ }^{8}$

Results from a cross-national survey providing insight into the impact of dry eye symptoms on patients' perspectives and experience of disease are reported in a parallel publication. $^{12}$ The study involved initial qualitative phone call interviews to optimise the survey instrument, followed by a quantitative online questionnaire administered to 160 patients with moderate-to-severe dry eye symptoms.

Herein, we examine the differences in patient preference and needs between respondents with moderate and severe DED based on their symptoms, as well as comparing preference scores of patients across the four participating countries: Australia, Germany, the UK and the USA.

\section{MATERIALS AND METHODS}

Overview of main steps

The methodology used in the patient survey is detailed elsewhere ${ }^{12}$ and will only be briefly summarised here. Following an initial literature review and social media listening analysis, in-depth telephone interviews were carried out with 12 patients ( 3 per country). The results were discussed with a patient association (the German Association for the Blind and Visually Impaired (DBSV)) and reviewed by the authors, who helped shape the questionnaire for the quantitative survey.

The online quantitative questionnaire involved a selfexplicated conjoint methodology using successive ratings of three layers of the survey features: levels, attributes and domains. $^{12} 13$

\section{Patient sample}

The study included patients with DED selected by symptoms using standard questionnaires from a PRO instrument and self-reported diagnosis. Patient selection for the online survey was conducted through a recruitment screener, which was validated by the authors and included questions about patients' DED diagnosis and medication use as prescribed by their eye doctor. Eligible patients received the 'symptom bother' module of the Impact of Dry Eye on Everyday Life (IDEEL) PRO Questionnaire to fill out. This module is a validated diagnostic tool used in clinical practice and as part of clinical studies. ${ }^{14}$ The designation of 'moderate' or 'severe' disease relied solely on symptomatology, as defined by the IDEEL. On this basis, at least 15 moderate patients (IDEEL symptom bother score 39-65) and at least 15 severe patients (score $\geq 66$ ), as determined based on their symptomology, were selected per country. Inclusion and exclusion criteria are summarised in online supplementary table 1 .

\section{Ethics, approval exemption and code of conduct adherence}

As an online patient survey, the study was conducted in accordance with the codes of both British Healthcare Business Intelligence Association and European Pharmaceutical Market Research Association, which ensure that this type of research is based on sound scientific questions. Adequate data protection statements were also included and participants' personal data were protected. The patients were entitled to withdraw at any stage of the process or could request that part or all of the record of their interview was destroyed or deleted. The study was granted approval by the Heartland Institutional Review Board, Illinois, USA.

This online quantitative survey was completed via a secure website, with all information protected from external sources. Patients received financial compensation for their voluntary participation at fair market value in line with the country regulations.

\section{Patient involvement}

The preparatory work for this study included a social media listening analysis, which captures the patient experiences and needs in their own words. A patient support group for the blind in Germany (DBSV) was consulted through regular teleconferences at all stages of the project from design to execution, data analysis and reporting.

The draft survey research questions were formulated based on a prior literature review and the social media listening study. They were then reviewed and critiqued by the three international dry eye clinical experts consulting on this project, as well as by the consultant from DBSV, to ensure that patient perspectives were accurately captured. The revised research questions were used to finalise the online survey design.

Recruitment for this study was carried out using dry eye patient panels, which included reaching out to patient 
groups for this indication. A survey experience assessment was performed at the end of the study to address questions around the time required to complete the survey, ease of completion, ease of understanding and patients' interest in the survey topic.

\section{Survey responses and statistical analysis}

Patients considered the importance of four main aspects (domains) of dry eye: 'treatment satisfaction', symptom bother, 'treatment administration' and 'impact on daily life'. Within each domain, patients evaluated the importance of different attributes. Each attribute was then broken down into a number of levels, as detailed in an accompanying publication, ${ }^{12}$ and are included as part of the online supplement (online supplementary table 2).

To weight the levels within an attribute, respondents gave the most important level a score of 100 and rated the others with a score of $0-100$ relative to the most important level. They also weighted the attributes of each domain in the same manner, that is, by giving the most important attribute a score of 100 and scoring the others relative to this. For example, on the symptom bother domain, patients rated 'eye discomfort', 'eye pain', 'eye fatigue' and so on relative to each other. Finally, respondents were asked to weight the four domains relative to each other using the same approach. From previous self-explicated surveys, the typical standardised SD for responses to these rating-type questions is around $20 \%$. With a sample size of 40 , we expected a SE of around $3 \%$. This makes it unlikely that any significant differences in ratings were due to sampling errors.

QoL was assessed using the 5-level European Quality of Life-5 Dimensions (EQ-5D-5L) questionnaire, a preference-based, standardised measure comprising five dimensions: mobility, self-care, usual activities, pain/ discomfort and anxiety/depression. ${ }^{15}$

\section{RESULTS}

\section{Patient population}

The survey included 160 patients with moderate-tosevere DED based on their symptoms, equally split across the four participating countries (UK, Australia, Germany and USA), as described elsewhere. ${ }^{12}$ The study sample, which was based on a dry eye demographic, included $70 \%$ female respondents; $75 \%$ of participants were aged over 40 years (those aged below 20 or over 80 years were excluded). Demographic characteristics, including age, gender, time of last eye exam and other dry eye conditions, are presented in online supplementary figure 1.

\section{Treatment comparison between patients with moderate and severe DED}

The relative importance of each of the four domains, treatment satisfaction, symptom bother, treatment administration and impact on daily life is shown in figure 1. For patients with moderate DED $(n=83)$, treatment satisfaction was the most important domain (conjoint estimate value $(\mathrm{CEV}) 0.269, \mathrm{p}<0.001$ vs second most important

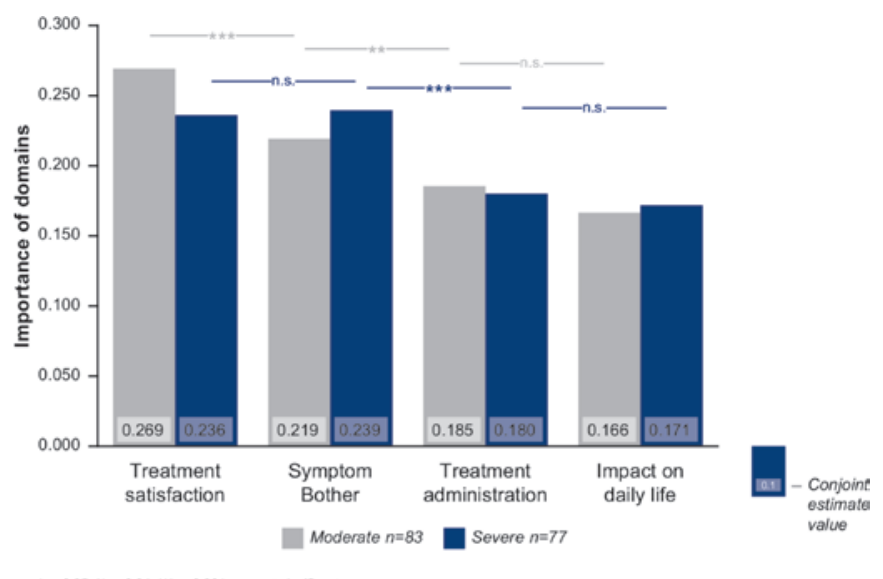

Figure 1 Level of importance of four main aspects (domains) of dry eye for patients with moderate and severe DED. DED, dry eye disease.

aspect). For patients with severe DED ( $\mathrm{n}=77)$, symptom bother was the most important domain (CEV 0.239).

The relative importance of attributes within each of the four domains, as provided by moderate versus severe patients, is displayed in figure 2A-D.

Within the treatment satisfaction domain, both groups classified 'treatment effectiveness on symptoms of dry eye' as the most important attribute (CEV 0.056 and 0.048 for moderate and severe patients, respectively $(\mathrm{p}<0.001$ vs third most important aspect)) (figure 2A). In both moderate and severe DED, patients felt that fast relief ('how quickly treatment starts to relieve symptoms') was an important attribute of treatment (CEV 0.049 and 0.038 for moderate and severe groups, respectively). 'Side effects of treatment' appeared to be less important for both moderate and severe dry eye patients (CEV 0.027 and 0.024, respectively). However, among the levels tested for this attribute (online supplementary table 2), 'stinging/burning/discomfort on instillation' was ranked as the most burdensome side effect.

With regards to symptom bother, patients with moderate DED considered eye discomfort to be the most important attribute (CEV 0.043, ( $\mathrm{p}<0.001$ vs second most important aspect)), whereas patients with severe DED rated eye sensitivity as the most bothersome symptom (CEV 0.047 vs 0.032 for moderate patients) (figure 2B). Among the levels tested for the eye discomfort attribute (online supplementary table 2), the most burdensome aspect was eye dryness, whereas for 'eye sensitivity' it was 'sensitivity due to wind or re-circulated air'.

On the treatment administration domain, patients with moderate disease rated 'frequency of treatment use' highest (CEV 0.054 vs 0.046 for severe patients), whereas for those with severe DED, the most important attribute was 'how the treatment works' (CEV 0.051 vs 0.048 for the moderate group) (figure 2C). Among the levels tested for how the treatment works (online supplementary table 2), patients preferred artificial tears, and for the "how the medicine is applied' attribute they selected 'drops/ 


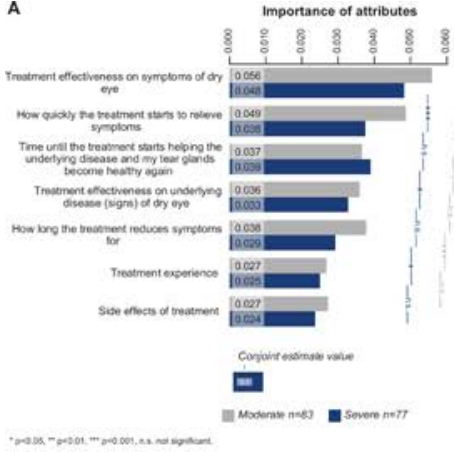

B

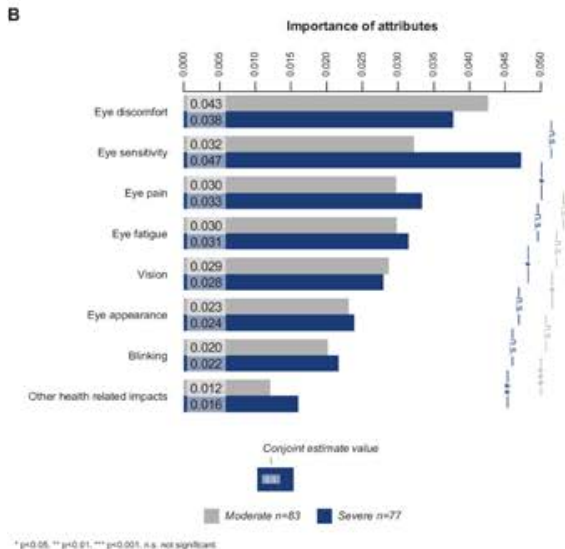

c

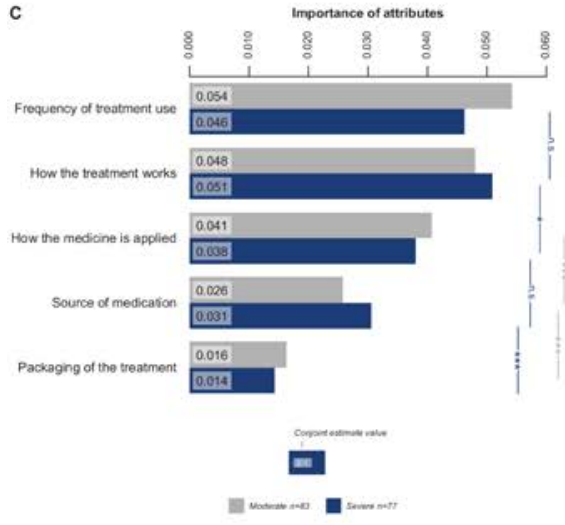

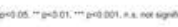

D

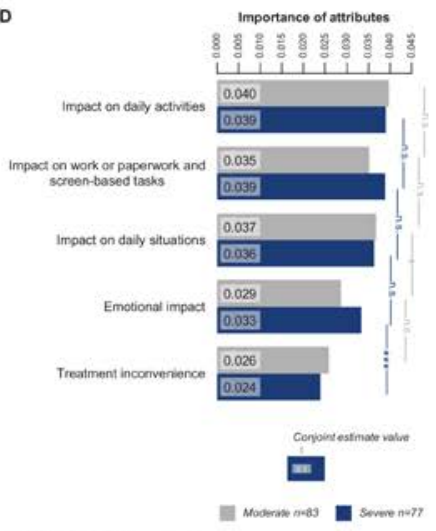

Figure 2 (A-D) Relative importance of attributes across the four domains of: 'treatment satisfaction' (A), 'symptom bother' $(B)$, 'treatment administration' $(C)$ and 'impact on daily life' (D). drops with a dropping aid' as the method of choice. With regards to frequency of treatment use, patients expressed a preference for 'as needed' over strict dosing schedules. 'Packaging of treatment' had the lowest score within this domain (online supplementary table 2).

With respect to impact on daily life, patients with moderate disease ranked 'impact on daily activities' highest (CEV 0.040), whereas for patients with severe DED both impact on daily activities and 'impact on work or paperwork and screen-based tasks' were rated as the most important (CEV 0.039) (figure 2D). Among the levels tested for the impact on daily activities attribute (online supplementary table 2), the highest score was assigned to 'difficulty working on a computer', whereas for 'impact on paper work and screen-based tasks' the most important aspect was 'feeling like you cannot focus'.

\section{Country comparison of patient preferences}

The relative importance scores for different aspects of DED were largely similar among the four participating countries (table 1). There was no preformulated hypotheses with respect to country differences in preference scores, so most of the country comparisons were not statistically significant.

Nevertheless, a number of country-specific differences emerged:

Patients in the USA gave significantly higher scores to 'treatment experience' ('moisturising or hydrating effect'; 64\% proportion of the highest value, CEV 0.031), compared with other countries $(\mathrm{p}<0.001)$. US patients also scored treatment side effects as more important compared with patients from other countries. By comparison with other countries, patients in Australia gave significantly lower scores to 'source of medication' (ie, prescription or non-prescription) (33\% proportion of the highest value, CEV 0.020; $\mathrm{p}<0.01$ ).

Patients in the UK rated most treatment-related aspects as highly important. They gave significantly higher scores to how the medicine is applied (80\% proportion of the highest value, CEV 0.045), compared with other countries $(p<0.001)$. By comparison with patients from other countries, respondents from Germany gave significantly lower scores to the attributes of 'vision' $(p=0.020)$ and 'eye appearance' $(\mathrm{p}=0.009)$ within the symptom bother domain, as well as to 'impact on daily situations' $(\mathrm{p}=0.013)$ within impact on daily life.

More country-specific results and comparisons are presented as part of the online supplementary information.

\section{Quality of life}

The average EQ-5D score for all patients included in the study was 0.713 and was mainly influenced by pain/ discomfort. The average EQ-5D scores were 0.764 and 0.658 in patients with moderate and severe dry eye symptoms, respectively. The average scores for patients in specific countries were: Germany, 0.790 ( 0.877 moderate patients, 0.718 severe patients); UK, 0.575 (0.652 


\begin{tabular}{|c|c|c|c|c|c|}
\hline & & Australia (\%) & Germany (\%) & UK (\%) & USA (\%) \\
\hline \multirow[t]{7}{*}{ Treatment satisfaction } & $\begin{array}{l}\text { Treatment effectiveness on symptoms } \\
\text { of dry eye }\end{array}$ & 100 & 100 & 84 & 100 \\
\hline & $\begin{array}{l}\text { How quickly the treatment starts to } \\
\text { relieve symptoms }\end{array}$ & 74 & 77 & 100 & 92 \\
\hline & $\begin{array}{l}\text { Time until the treatment starts helping } \\
\text { the underlying disease and my tear } \\
\text { glands become heathy again }\end{array}$ & 61 & 58 & 75 & 87 \\
\hline & $\begin{array}{l}\text { How long the treatment reduces } \\
\text { symptoms for }\end{array}$ & 52 & 55 & 66 & 81 \\
\hline & $\begin{array}{l}\text { Treatment effectiveness on underlying } \\
\text { disease (signs) of dry eye }\end{array}$ & 62 & 53 & 68 & 78 \\
\hline & Side effects of treatment & 37 & 42 & 44 & 67 \\
\hline & Treatment experience & 55 & 33 & 50 & 64 \\
\hline \multirow[t]{8}{*}{ Symptom bother } & Eye sensitivity & 91 & 100 & 81 & 81 \\
\hline & Eye discomfort & 100 & 72 & 100 & 100 \\
\hline & Eye pain & 69 & 59 & 73 & 90 \\
\hline & Vision & 70 & 53 & 67 & 69 \\
\hline & Eye fatigue & 71 & 53 & 86 & 77 \\
\hline & Eye appearance & 60 & 36 & 61 & 63 \\
\hline & Blinking & 50 & 36 & 46 & 61 \\
\hline & Other health-related impacts & 26 & 25 & 38 & 42 \\
\hline \multirow{5}{*}{$\begin{array}{l}\text { Treatment } \\
\text { administration }\end{array}$} & Frequency of treatment use & 100 & 100 & 86 & 69 \\
\hline & How the treatment works & 72 & 74 & 100 & 100 \\
\hline & How the medicine is applied & 69 & 71 & 80 & 72 \\
\hline & Source of medication & 33 & 58 & 58 & 56 \\
\hline & Packaging of the treatment & 22 & 27 & 24 & 35 \\
\hline \multirow[t]{5}{*}{ Impact on daily life } & $\begin{array}{l}\text { Impact on work or paperwork and } \\
\text { screen-based tasks }\end{array}$ & 90 & 100 & 93 & 86 \\
\hline & Impact on daily activities & 100 & 98 & 98 & 100 \\
\hline & Impact on daily situations & 94 & 87 & 100 & 90 \\
\hline & Emotional impact & 76 & 70 & 92 & 77 \\
\hline & Treatment inconvenience & 47 & 63 & 76 & 64 \\
\hline
\end{tabular}

moderate patients, 0.517 severe patients); USA, 0.777 (0.797 moderate patients, 0.755 severe patients); and Australia, 0.626 ( 0.759 moderate patients, 0.638 severe patients) (online supplementary figure 2 ).

With respect to impact on daily life, more patients with severe disease believed that dry eye symptoms had a strong or extreme impact compared with moderate DED patients. The most burdensome aspects of DED on patients' life were discomfort and limitations of daily activities. Among the levels tested for impact on daily situations (online supplementary table 2), patients thought that 'difficulty going outdoors in windy conditions' was the most important aspect, whereas for 'emotional impact' it was the 'worry that their eyes would get worse'. Respondents with severe DED scored higher than moderate patients on all levels of emotional impact, except for 'feeling different from other people because of dry eyes'.

\section{DISCUSSION}

\section{Clinical context}

It is increasingly recognised that understanding patients' views and preferences can support drug development and clinical decision-making by improving the fit between products and patient needs. ${ }^{816}$ In DED, efforts are being made to better capture the patient-reported dry eye symptoms and their impact on QoL, and to develop methodologies that assess and monitor these outcomes in drug development studies and clinical practice. ${ }^{17}$

A recent review examining QoL and treatment satisfaction concluded that patient-reported symptoms should be integrated into the individualised approach to treatment 
in DED. ${ }^{18}$ Given the unmet need for adequate endpoints in clinical studies of DED, ${ }^{4}$ patient-based evidence can also provide valuable information for clinical trial design. ${ }^{8}$ However, despite an increased awareness of the need for patient preference studies in DED, the published literature in this area is limited.

Country-specific data on the patient experience of DED are particularly sparse. Nevertheless, a recent cross-sectional survey of 706 patients with DED from five European countries (France, Germany, Italy, Spain and the UK) provided some insight into regional perspectives. ${ }^{19}$ In this survey, more patients in France considered DED to be a 'handicap' (21\%) compared with the overall percentages for all countries $(12 \%, \mathrm{p}<0.05)$. Meanwhile, only $11 \%$ and $8 \%$ of UK patients considered DED a handicap and a disease, respectively.

Our study adds to the current efforts to integrate patient preference into the broader clinical management of DED by providing insight into the perspectives of patients with different degrees of symptom severity, as well as regional differences in patient priorities and needs.

\section{Impact of DED severity on patients' experience of disease}

As expected, the preferences of patients with different degrees of symptom severity were similar in many respects. Given the progressive nature of the disease, the moderate and severe groups identified in this study lie on a population continuum, whereby patients' needs and preferences may differ as the disease severity progresses. Hence, on certain attributes of dry eye, our survey found clear differences in relation to the greater symptom burden experienced by patients in the more advanced stages of disease.

Thus, patients with moderate DED scored the importance of treatment satisfaction significantly higher than those with severe disease $(p<0.01)$. In particular, treatment effectiveness was perceived as very important by patients with moderate DED, and this group also gave significantly higher scores to attributes such as 'how quickly the treatment starts to relieve symptoms' and 'how long the treatment reduces symptoms for', compared with severe patients $(\mathrm{p}<0.01)$. By contrast, symptom bother was scored as the most important domain by patients classified as having severe DED, possibly because the currently available treatments are less likely to alleviate dry eye symptoms in this more severe group. Within the symptom bother domain, patients with severe DED indicated a greater importance for parameters such as: eye sensitivity (sensitivity due to wind or recirculated air and sensitivity due to light or glare), eye pain (burning eyes, stinging eyes and general eye pain), eye fatigue (need to close your eyes), eye appearance (excessive eye watering or discharge) and other health-related symptoms (such as allergy-like symptoms and headaches) than patients with moderate disease. This is consistent with recent findings indicating a greater sensitivity to neuropathic-like ocular pain in patients with moderate-to-severe dry eye and ocular itch symptoms. ${ }^{20}$

These results reinforce the considerable health burden posed by severe $\mathrm{DED}^{21}$ due to symptoms affecting sufferers' physical and visual appearance and functioning, pain and non-DED-specific aspects. The impact of DED on patients' emotional well-being was also highlighted in the social media listening project preceding this survey, which found that around $1 \%$ of patients with dry eye mentioned suicidal tendencies in their online posts. ${ }^{22}$ These perceptions were echoed in our QoL analysis, in which the EQ-5D score obtained for all patients (0.713) positions the burden of DED between that of diabetes mellitus $\left(0.751^{23}\right)$ and multiple sclerosis before relapse $\left(0.648^{24}\right)$. Results of the EQ-5D-5L questionnaire are important as they enable the comparison of HRQoL impact of patients suffering from different conditions in relation to their expressed preferences, as could also be determined as part of a recent patient preference study with non-alcoholic steatohepatitis patients. ${ }^{25}$ In the present study, the QoL burden was particularly high in patients with severe DED, which had an average EQ-5D 5 L score of 0.658. For comparison, a US report of preference-based EQ-5D scores for chronic conditions found that the mean score of a healthy 51-year olds (the average age of respondents in the present study) was 0.842; 0.834 for patients with psoriasis; 0.695 for angina; 0.636 for heart failure; and 0.651 for renal failure. ${ }^{23}$

Previous patient preference surveys also found that severe DED could reduce QoL to a considerable extent, which was comparable to severe angina and disabling hip fracture. $^{326}$

With regards to patients' needs from treatment, respondents with severe dry eye symptoms placed more importance on therapeutic mechanism of action than moderate patients, probably because they had developed a greater awareness of the persistent nature of their condition and the need to address its underlying causes. These patients acknowledged that treating dry eye might require longer times, whereas moderate patients viewed DED predominantly as an inconvenience and required immediate symptom relief from their treatment.

The need for effective therapeutic options in moderate-to-severe DED is well acknowledged by treating physicians. In a survey of 235 ophthalmologists and corneal specialists, only $33 \%$ of respondents thought that current therapies were very effective for moderate DED and only $5 \%$ for severe disease. ${ }^{3}$

\section{Country-specific considerations}

The four participating countries showed similar results across the domains examined. All patients thought DED had a considerable impact on their daily activities, work-related tasks and daily situations, and $70 \%-92 \%$ of patients across the four countries scored the emotional impact of disease as high. Some differences in patient preference emerged on analysis of country-specific variance. For example, patients from Australia ranked 
treatment experience as significantly more important than the concern of side effects, whereas respondents from Germany exhibited the opposite trend $(p<0.05$ for both). Slight differences between countries also existed in the patient ranking of bothersome symptoms such as eye discomfort, eye pain, eye sensitivity and eye fatigue.

\section{Treatment modalities}

With respect to treatment-related aspects, patients from all four countries preferred their treatment in the form of artificial tears and preferred drops/drops with a dropping aid as method of application. This could be attributed to the immediate benefit provided by symptomatic treatment with artificial tears (eg, lubricating effect) compared with the slower onset of effect from potential disease-modifying treatments devoid of an immediate lubricating effect on the eye.

Patients in Australia, Germany and the USA preferred their artificial tears in the form of eye drops in a preservative-free formulation. They wanted to use their medication as needed and were not concerned with the source of the eye drops. Patients in the UK expressed a preference for preservative-free artificial tears in the form of a spray, prescribed by a doctor and used as needed.

Of note, the current experience with disease-modifying agents is very limited in Australia, Germany and UK, by comparison with the USA. In Germany and UK, cyclosporine A has been available since 2015, but its mechanism of action, time of onset and side effects are not well known to patients compared with those in the US, underscoring the need for patient education about newly available therapeutic options. Despite these differences, artificial tears were also the preferred option for US respondents, highlighting the patients' desire for adequate and immediate symptom relief. This need was confirmed by patients' ranking of 'treatment effectiveness on DED symptoms' as one of the most important attributes in all countries. Surprisingly, the source of medication (prescription or non-prescription) was not as important for many respondents, despite the fact that prescription drugs are most often reimbursed.

Our cross-national quantitative survey provides insights into the perspectives and experiences of dry eye patients from a wide range of settings, and highlights the differences between patients with moderate and severe dry eye symptoms, as well as between patients from different countries. The study sample is geographically heterogeneous and representative of the moderate-to-severe DED patient population through the use of a screening questionnaire. However, the study methodology may have influenced patient selection to some extent, as patients who are uncomfortable looking at screens, or those with a limited interest in their condition, may not have opted to enrol in this type of online survey. The disease/ symptom history of individual respondents and their treatment experience might have impacted responses, making comparisons difficult. Finally, the potential impact of comorbid conditions on the expressed needs and preferences of the DED patients was not solicited, and therefore not captured, in this study.

In summary, the results presented herein confirm that severe DED poses a significant health burden for sufferers from all four countries and has a major impact on their QoL. The patient ranking of symptoms and treatment attributes helps identify the needs and expectations of dry eye patients and can inform treatment development and decisions.

Acknowledgements The authors wish to thank all the survey participants for their involvement in the study.

Contributors All authors contributed to the development of the study concept, data analysis and interpretation. With regard to thedesign of this study, the authors were consulted by the Deutscher Blinden und Sehbehindertenverband e.V. (DBSV) as regulated by a contract between Novartis and DBSV. All authors meet the International Committee of Medical Journal Editors (ICMJE) criteria for authorship for this article and take responsibility for the integrity of the work as a whole. All authors were involved in the preparation and reviewof the manuscript and approved the final version to be submitted.

Funding The sponsorship of this study and article processing charges were funded by Novartis Pharma AG, Basel, Switzerland. All authors had full access to all of the data in this study and take complete responsibility for the integrity of the data and accuracy of the data analysis. All named authors meet the International Committee of Medical Journal Editors (ICMJE) criteria for authorship for this article, take responsibility for the integrity of the work as a whole and have given their approval for this version to be published. With regard to the design of this study, the authors were consulted by the Deutscher Blinden und Sehbehindertenverband e.V. (DBSV) as regulated by a contract between Novartis and DBSV. Anna Panpurina from InPharmation also provided her assistance with the design of the study and the interpretation of the data. Writing assistance in the preparation of this article was provided by lleana Stoica, Marie-Catherine Mousseau and lain Hatch (Novartis Ireland). Support for this assistance was funded by Novartis.

Competing interests EM: Consultant for Alcon, Dompe, Pharm Allergan, Santen $\mathrm{GmbH}$, Shire, Sun Pharmaceuticals, Thea GmbH, Visufarma. Lecturer for Alcon, Dompe, Pharm Allergan, Santen $\mathrm{GmbH}$, Thea $\mathrm{GmbH}$, Ursapharm $\mathrm{GmbH}$, Visufarma. CC: Consultant for Alcon and has participated in advisory boards by Alcon/Novartis and Shire. PA has received research funding from Alcon and MC2 Therapeutics and has participated in advisory boards by Alcon/Novartis, Allergan, and Shire. GJ is employee of InPharmation. NC and BS are employees of Novartis. In addition to his role as employee of Novartis, NC is a member of IMI PREFER and is also involved with working groups on patient preferences.

Patient consent for publication Not required.

Ethics approval Our study obtained approval from the Heartland Institutional Review Board and the research conformed to the codes of both BHBIA and EphMRA. These codes of conduct stop this type of research being used as a front for marketing activities and ensure that all adverse event procedures are properly adhered to. The patient cooperation was voluntary, and they were entitled to withdraw at any stage of the process, or subsequently, to ask that part or all of the record of their interview was destroyed or deleted. The patients were advised that the information they provided was for market research purposes only, and would be treated confidentially, without reference to individual names. They were assured that any information they give would simply be added to the answers received from other respondents to provide an overall picture of views, which may be used in a scientific publication.

Provenance and peer review Not commissioned; externally peer reviewed.

Data availability statement Data may be obtained from a third party and are not publicly available.

Open access This is an open access article distributed in accordance with the Creative Commons Attribution Non Commercial (CC BY-NC 4.0) license, which permits others to distribute, remix, adapt, build upon this work non-commercially, and license their derivative works on different terms, provided the original work is properly cited, appropriate credit is given, any changes made indicated, and the use is non-commercial. See: http://creativecommons.org/licenses/by-nc/4.0/.

Nigel Cook http://orcid.org/0000-0001-8211-7613 


\section{REFERENCES}

1 Craig JP, Nichols KK, Akpek EK, et al. TFOS DEWS II definition and classification report. Ocul Surf 2017;15:276-83.

2 Messmer EM. The pathophysiology, diagnosis, and treatment of dry eye disease. Dtsch Arztebl Int 2015;112:71-82.

3 Asbell PA, Spiegel S. Ophthalmologist perceptions regarding treatment of moderate-to-severe dry eye: results of a physician survey. Eye Contact Lens 2010;36:33-8.

4 Roy NS, Wei Y, Kuklinski E, et al. The growing need for validated biomarkers and endpoints for dry eye clinical research. Invest Ophthalmol Vis Sci 2017;58:BIO1-19.

5 Schiffman RM, Walt JG, Jacobsen G, et al. Utility assessment among patients with dry eye disease. Ophthalmology 2003;110:1412-9.

6 Patel VD, Watanabe JH, Strauss JA, et al. Work productivity loss in patients with dry eye disease: an online survey. Curr Med Res Opin 2011;27:1041-8.

7 Craig BM, Lancsar E, Mühlbacher AC, et al. Health preference research: an overview. Patient 2017;10:507-10.

8 Cook NS, Cave J, Holtorf A-P. Patient preference studies during early drug development: aligning stakeholders to ensure development plans meet patient needs. Front Med 2019;6.

9 Bywall KS, Veldwijk J, Hansson MG, et al. Patient perspectives on the value of patient preference information in regulatory decision making: a qualitative study in Swedish patients with rheumatoid arthritis. Patient 2019;12:297-305.

10 de Bekker-Grob EW, Berlin C, Levitan B, et al. Giving patients' preferences a voice in medical treatment life cycle: the prefer publicprivate project. Patient 2017;10:263-6.

11 van Overbeeke E, Whichello C, Janssens R, et al. Factors and situations influencing the value of patient preference studies along the medical product lifecycle: a literature review. Drug Discov Today 2019;24:57-68.

12 Asbell PA, Messmer EM, Chan C, et al. Defining the needs and preferences of patients with dry eye disease. BMJ Open Ophthalmol.

13 Sattler HBS. A comparison of conjoint measurement with selfexplicated approach. In: Gustafsson A, Herrmann A, Hubert H, eds. Conjoint measurement: methods and application. Berlin: Springer Verlag, 2000: 121-3.
14 Abetz L, Rajagopalan K, Mertzanis P, et al. Development and validation of the impact of dry eye on everyday life (IDEEL) questionnaire, a patient-reported outcomes (pro) measure for the assessment of the burden of dry eye on patients. Health Qual Life Outcomes 2011;9:111.

15 Herdman M, Gudex C, Lloyd A, et al. Development and preliminary testing of the new five-level version of EQ-5D (EQ-5D-5L). Qual Life Res 2011;20:1727-36.

16 Allen JD, Stewart MD, Roberts SA, et al. The value of addressing patient preferences. Value Health 2017;20:283-5.

17 Barabino S, Labetoulle M, Rolando M, et al. Understanding symptoms and quality of life in patients with dry eye syndrome. Ocul Surf 2016;14:365-76.

18 Gomes JAP, Santo RM. The impact of dry eye disease treatment on patient satisfaction and quality of life: a review. Ocul Surf 2019;17:9-19.

19 Labetoulle M, Rolando M, Baudouin C, et al. Patients' perception of DED and its relation with time to diagnosis and quality of life: an international and multilingual survey. $\mathrm{Br} J$ Ophthalmol 2017;101:1100-5.

20 Galor A, Small L, Feuer W, et al. The relationship between ocular itch, ocular pain, and dry eye symptoms (an American Ophthalmological Society thesis). Trans Am Ophthalmol Soc 2017;115:T5.

21 Uchino M, Schaumberg DA. Dry eye disease: impact on quality of life and vision. Curr Ophthalmol Rep 2013;1:51-7.

22 Cook N, Mullins A, Gautam R, et al. Evaluating patient experiences in dry eye disease through social media listening research. Ophthalmol Ther 2019;8:407-20.

23 Sullivan PW, Ghushchyan V. Preference-based EQ-5D index scores for chronic conditions in the United States. Med Decis Making 2006;26:410-20.

24 Orme M, Kerrigan J, Tyas D, et al. The effect of disease, functional status, and relapses on the utility of people with multiple sclerosis in the UK. Value Health 2007;10:54-60.

25 Cook N, Geier A, Schmid A, et al. The patient perspectives on future therapeutic options in NASH and patient needs. Front Med 2019;6.

26 Buchholz P, Steeds CS, Stern LS, et al. Utility assessment to measure the impact of dry eye disease. Ocul Surf 2006;4:155-61. 